\title{
miR-21 synergizes with BMP9 in osteogenic differentiation by activating the BMP9/Smad signaling pathway in murine multilineage cells
}

\author{
QILING SONG, LIANG ZHONG, CHU CHEN, ZUCHUAN TANG, HONGXIA LIU, YIQIN ZHOU, MIN TANG, \\ LAN ZHOU, GUOWEI ZUO, JINYONG LUO, YAN ZHANG, QIONG SHI and YAGUANG WENG
}

Key Laboratory of Diagnostic Medicine designated by the Chinese Ministry of Education, Chongqing Medical University, Chongqing 400016, P.R. China

Received June 22, 2015; Accepted September 30, 2015

DOI: $10.3892 /$ ijmm.2015.2363

\begin{abstract}
Bone morphogenetic proteins (BMPs), particularly BMP9, have been shown to promote the osteogenic differentiation of murine multilineage cells (MMCs) and to promote bone formation in bone diseases; however, the mechanisms involved remain poorly understood. MicroRNAs (miRNAs or miRs) have been proven to regulate mesenchymal stem cell (MSC) differentiation. In this study, we identified a novel mechanism that unravels the functional axis of a key miRNA (miR-21) which contributes to BMP9-induced osteogenic differentiation. We screened differentially expressed miRNAs in MMCs during BMP9-induced osteogenic differentiation and found that miR-21 was significantly upregulated by BMP9 during the osteogenesis of MMCs. Furthermore, miR-21 was confirmed to promote the osteogenic differentiation of the MMCs by suppressing Smad7, which negatively regulates the osteogenic differentiation of MMCs. The upregulation of miR-21 may promote the osteogenic differentiation of MMCs in synergy with BMP9. The findings of our study revealed a novel function of miR-21, and suggest that the overexpression of miR-21 contributes to bone formation by promoting BMP9-induced osteogenic differentiation. Our data may provide a molecular basis for the development of novel therapeutic strategies to treat bone diseases, such as osteoporosis and other inflammatory bone diseases.
\end{abstract}

\section{Introduction}

Bone remodeling is regulated by both the number and activity of osteoblasts and osteoclasts. Osteoblast lineage commitment,

Correspondence to: Professor Yaguang Weng or Dr Qiong Shi, Key Laboratory of Diagnostic Medicine designated by the Chinese Ministry of Education, Chongqing Medical University, 1 Yixueyuan Road, Yuzhong, Chongqing 400016, P.R. China

E-mail: yaguangweng@126.com

E-mail: anniesq8718@aliyun.com

Key words: miR-21, bone morphogenetic protein 9, Smad7, osteogenic differentiation proliferation and differentiation are controlled by a welldefined genetic program. MicroRNAs (miRNAs or miRs) are non-coding RNAs, 22 nucleotides in length that are involved in the regulation of gene expression, coordinating a broad spectrum of biological processes (1). In mammals, the active strand miRNA sequence of $\sim 22$ nucleotides partially recognizes the complementary miRNA recognition element (MRE) or seed-matched sequences located mostly in the 3'-untranslated region (UTR) of mRNAs $(2,3)$. miRNAs have emerged as important post-transcriptional regulators of gene expression (4-9). Aberrant miRNA destabilization is associated with various developmental abnomalities and human diseases. The elevated expression of miR-21 has been observed in multiple types of tumors, and the suppression of miR-21 in tumors has been shown to lead to reduced cell proliferation and migration, suggesting that miR-21 acts as an oncogene (10-19).

Several studies have focused on miRNAs being modulated by bone morphogenetic protein (BMP) signaling as a means to demonstrate the role of miRNAs in osteoblasts $(9,20,21)$. BMP7 was identified as a direct target of miR-542-3p; it was observed that the overexpression of miR-542-3p suppresses BMP7 and inhibits BMP7/phosphoinositide 3-kinase (PI3K)survivin signaling. However, the inhibition of miR-542-3p by anti-miR-542-3p led to increased bone formation (22). miR-21 has been shown to modulate the extracellular signal-regulated kinase (ERK)-mitogen-activated protein kinase (MAPK) signaling pathway by suppresing sprouty homolog 2 (Drosophila) (SPRY2) expression during mesenchymal stem cell (MSC) differentiation (23). Another study indicated that suppressing the expression and function of miR-21 may contribute to the tumor necrosis factor- $\alpha$ (TNF- $\alpha$ )-induced inhibition of bone formation in estrogen deficiency-induced osteoporosis (24).

There are at least 14 BMPs in mammalian cells. BMP2, BMP4, BMP6, BMP7 and BMP9 have been shown to induce MSC differentiation into osteoblasts. We have previously demonstrated that BMP9 is one of the most potent osteogenic BMPs (25). Therefore, the role of miR-21 in BMP9-induced osteogenic differentiation is of interest for the development of therapies for bone fractures and bone diseases, such as postmenopausal osteoporosis. 
In the present study, a key miRNA (miR-21) was upregulated in murine multilineage cells (MMCs) by BMP9. We hypothesized that the upregulation of miR-21 may activate the BMP9/Smad signaling pathway to sustain BMP9/Smad activation during MMC osteogenic differentiation.

\section{Materials and methods}

Cell lines and culture. The HEK-293 (human embryonic kidney), HCT116 (colorectal carcinoma) and C2C12 (murine myoblasts) cell lines, and mouse embryonic fibroblasts (MEFs) were obtained from the American Type Culture Collection (ATCC; Manassas, VA, USA). The HEK-293 cells were maintained in complete Dulbecco's modified Eagle's medium (DMEM) supplemented with $10 \%$ fetal calf serum (FCS) (HyClone, Logan, UT, USA), $100 \mathrm{U} / \mathrm{ml}$ penicillin and $100 \mu \mathrm{g} / \mathrm{ml}$ streptomycin at $37^{\circ} \mathrm{C}$ in $5 \% \mathrm{CO}_{2}$. The $\mathrm{C} 2 \mathrm{C} 12$ and HCT116 cells and MEFs were maintained in DMEM, supplemented with $10 \%$ fetal bovine serum (FBS) (Gibco, Grand Island, NY, USA), $100 \mathrm{U} / \mathrm{ml}$ penicillin and $100 \mu \mathrm{g} / \mathrm{ml}$ streptomycin at $37^{\circ} \mathrm{C}$ in $5 \% \mathrm{CO}_{2}$.

Oligoribonucleotide transfection assay. Oligoribonucleotides for the regulation of miRNA expression were obtained from GenePharma (Shanghai, China). mmu-miR-21 mimics (miR-21) were simple-stranded RNA oligonucleotides with similar biological functions to mature endogenous miR-21, and mmu-miR-21 inhibitors (anti-miR-21) were designed to downregulate endogenous miR-21 expression. The sequences for each oligonucleotide are listed in Table I. Both oligonucleotides had their own negative control (NC). Entranster ${ }^{\mathrm{TM}}$ R4000 (Engreen Biosystems, Beijing, China) was utilized according to the manufacturer's instructions. The transfection assay was conducted based on a previously described protocol (26).

The $\mathrm{C} 2 \mathrm{C} 12$ cells and MEFs were transfected with $100 \mathrm{nM}$ of either a non-targeting small RNA oligonucleotide as a negative control (miR.NC) or miR-21 mimic, or mmu-miR-21 inhibitor in a well in a 24 -well plate or $500 \mathrm{nM}$ of the above in a well in a 6 -well plate. The transfection efficiency was determined under a fluorescence microscope.

Preparation of BMP9-conditioned medium. BMP9 and conditioned media were prepared as previously described (27). Briefly, subconfluent HCT116 cells (in $75-\mathrm{cm}^{2}$ flasks) were infected with an optimal titer of Ad-BMP9 or Ad-GFP control. At 8 h posttransfection, the culture medium was changed to serum-free DMEM. The conditioned medium was collected $48 \mathrm{~h}$ posttransfection and used immediately. The $\mathrm{C} 2 \mathrm{C} 12$ cells and MEFs were treated with Ad-BMP9- or Ad-GFP-conditioned medium for 9 days. As a blank group, cells were treated with conditioned medium without Ad-BMP9 or Ad-GFP and the other teatment conditions were same as those for the Ad-BMP9 or Ad-GFP groups.

Reverse transcription-polymerase chain reaction (RT-PCR). Total RNA was isolated from the cells using TRIzol reagent (Ambion, Aukland, New Zealand) according to the manufacturer's instructions for the analysis of mRNA and miRNA expression. The RNA concentration and purity were quantified using a spectrophotometer (NanoDrop 1000
Spectrophotometer; Thermo Fisher Scientific Inc., Waltham, MA, USA). The isolated RNA was used for successive experiments. Total RNA $(2,000 \mathrm{ng})$ was reverse transcribed using reverse transcription primers according to the manufacturer's instructions (Reverse Transcriptase M-MLV, Takara Code: D2639A, Takara Bio Inc., Otsu, Japan). The primers used for RT-PCR and quantitative PCR (qPCR; RT-qPCR) are listed in Table II. The specificity of the PCR products was confirmed by resolving PCR products on $1 \%$ agarose gels. The results were recorded using the Bio-Rad Electrophoresis Documentation system (GelDoc 1000; Bio-Rad Laboratories, Hercules, CA, USA) and Quantity One software, version 4.5.0.

Quantitative (real-time) PCR. cDNA was utilized as a template to amplify target genes, along with SYBR Premix Ex Taq (Takara Bio Inc.). Each RNA sample was evaluated in triplicate. Gene expression results were analyzed using the $\Delta \Delta \mathrm{Ct}$ method, and miR-21 was normalized to U6 and Smad7 to $\beta$-actin expression. qPCR was performed on a Bio-Rad iQ5 instrument (Bio-Rad, Hercules, CA, USA). Data were analyzed using Optical System software, version 2.0.

Alkaline phosphatase (ALP) staining. ALP staining was monitored using a Fast Violet B Salt kit (procedure no. 85; SigmaAldrich, St. Louis, MO, USA), as previously described (28). Briefly, we seeded the C2C12 cells and MEFs in 24-well cell culture plates and treated them with GFP-conditioned medium (CM), BMP9-CM, miR.NC + BMP9-CM, miR-21 + BMP9-CM, or anti-miR-21 + BMP9-CM for 7 days. One Fast Violet B Salt capsule (FBS25-10CAP) was dissolved in $48 \mathrm{ml}$ distilled water and $2 \mathrm{ml}$ naphthol AS-MX phosphate alkaline solution (N4875) (both from Sigma-Aldrich). The cells were fixed by immersing them in a citrate-buffered acetone solution ( 2 parts citrate to 3 parts acetone) for $30 \mathrm{sec}$ and were then rinsed in deionized water for $45 \mathrm{sec}$. The samples were then placed in the dark to undergo ALP staining for $30 \mathrm{~min}$. After 2 min of rinsing in deionized water, the cells were treated with Mayer's hematoxylin solution for $10 \mathrm{~min}$. Cells were scanned at both a lower [cells in a 24-well plate were scanned under a scanner (ScanMaker 3600; Shanghai Microtek Technology Co., Ltd., Shanghai, China)] and higher magnification (cells scanned under a bright field microscope, x200).

Alizarin red $S$ staining. We seeded the $\mathrm{C} 2 \mathrm{C} 12$ cells and MEFs in 24-well cell culture plates and treated them with GFP-CM, BMP9-CM, miR.NC + BMP9-CM, miR-21 + BMP9-CM, or anti-miR-21 + BMP9-CM for 14 days. The cells were fixed in $0.05 \%$ ice-cold glutaraldehyde for $10 \mathrm{~min}$ and rinsed with double-distilled $\mathrm{H}_{2} \mathrm{O}$. The cells were then stained with $40 \mathrm{mM}$ Alizarin red S (Sigma-Aldrich), $\mathrm{pH} 4.0$, for 15 min with gentle agitation. The cells were rinsed 5 times with double-distilled $\mathrm{H}_{2} \mathrm{O}$ and then rinsed for 15 min with $1 \mathrm{X}$ phosphate-buffered saline (PBS) while being gently agitated. Cells were scanned at both a lower [cells in a 24-well plate were scanned under a scanner (ScanMaker 3600; Shanghai Microtek Technology Co., Ltd.)] and higher magnification (cells scanned under a bright field microscope, x200).

miRNA target site prediction. The target prediction tools, TargetScan (http://www.targetscan.org), PicTar (http://pictar. 
Table I. Oligoribonucleotide information.

Sense $\left(5^{\prime} \rightarrow 3^{\prime}\right)$

Antisense $\left(5^{\prime} \rightarrow 3^{\prime}\right)$

mmu-miR-21

mimic (miR-21)

mmu-miR-21

inhibitor (anti-miR-21)

Negative control (NC)

Inhibitor $\mathrm{NC}$

Mimic NC

Stable NC
UAGCUUAUCAGACUGAUGUUGA

UCAACAUCAGUCUGAUAAGCUA

UUC UCC GAA CGU GUC ACG UTT

CAGUACUUUUGUGUAGUACAA

UUGUACUACACAAAAGUACUG

UUC UCC GAA CGU GUC ACG UTT
ACG UGA CAC GUU CGG AGA ATT

ACG UGA CAC GUU CGG AGA ATT

Table II. Primers used for RT-qPCR.

Gene symbol Sequence $\left(5^{\prime} \rightarrow 3^{\prime}\right)$

$\begin{array}{ll}\text { mmu-miR-21 } & \text { (F) TGGCGTAGCTTATCAGACTGA } \\ \text { U6 } & \text { (F) CTCGCTTCGGCAGCACATATACT } \\ \text { Mouse OCN } & \text { (F) TCTGACAAAGCCTTCATGTCC } \\ \text { Mouse Smad7 } & \text { (F) AAGATCGGCTGTGGCATC } \\ \text { Mouse } \beta \text {-actin } & \text { (F) CCTGAGGCTCTTTTCCAGCC } \\ \text { Mouse GAPDH } & \text { (F) ACCCAGAAGACTGTGGATGG }\end{array}$

(R) GTGCAGGGTCCGAGGT

(R) ACGCTTCACGAATTTGCGTGTC

(R) AAATAGTGATACCGTAGATGCG

(R) CCAACAGCGTCCTGGAGT

(R) TAGAGGTCTTTACGGATGTC

(R) CACATTGGGGGTAGGAACAC

OCN, osteocalcin; F, forward; R, reverse; GAPDH, glyceraldehyde 3-phosphate dehydrogenase.

Table III. Smad7 mRNA 3'-UTR binding to miR-21 sequence.

\begin{tabular}{ll}
\hline Gene name & Sequence $\left(5^{\prime} \rightarrow 3^{\prime}\right)$ \\
\hline Smad7 wild-type & (F) CTAGGCTCAATGAGCATGTTTAGAATTTAACATAAGCTATTTTTCTAACTACAAAGG \\
& (R) AGCTCCTTTGTAGTTAGAAAAATAGCTTATGTTAAATTCTAAACATGCTCATTGAGC \\
Smad7 mutant-type & (F) CTAGGCTCAATGAGCATGTTTAGAATTTAACATATCGAATTTTTCTAACTACAAAGG \\
& (R) AGCTCCTTTGTAGTTAGAAAAATTCGATATGTTAAATTCTAAACATGCTCATTGAGC
\end{tabular}

F, forward; R, reverse.

mdc-berlin.de/) and miRanda (http://www.microrna.org), were utilized in order to identify possible target genes of miR-21 in BMP9-induced osteogenic differentiation. Computational target prediction was primarily based on the potential pairing of the miRNA seed sequence to a complementary site in the 3'-UTR of a target mRNA, according to specific base-pairing rules.

Smad7 3'-UTR cloning and luciferase assay. Smad7 mRNA 3'-UTRs containing one miR-21-binding sequence for the mouse Smad7 gene (NCBI reference sequence: NM_001042660.1) were acquired by oligonucleotide synthesis following the manufacturer's instructions (pMIR-REPORT ${ }^{\mathrm{TM}}$ System, miRNA Expression Reporter Vector, Part No. AM5795, Ambion, Shanghai, China) (Table III). The annealing fragment was then subcloned into the SpeI site and HindIII site in the pMIR-REPORT ${ }^{\mathrm{TM}}$ miRNA expression reporter empty vector (Ambion, Shanghai, China). Binding-region mutations were achieved using an oligo synthesis following the manufacturer's instructions (Ambion). Transient transfection into HEK-293 cells $\left(1 \times 10^{4}\right.$ cells/well) was carried out in 24 -well plates with Lipofectamine $^{\mathrm{TM}} 2000$ (Invitrogen, Shanghai, China) following the manufacturer's instructions. The cells were co-transfected with $200 \mathrm{ng}$ of the luciferase construct plasmid and $50 \mathrm{ng}$ of pMIR-REPORT $\beta$-gal (Ambion) plasmid, and luciferase assays were performed using the dual-luciferase reporter assay system (Ambion) according to the manufacturer's instructions. Luminescent signals were quantified using a luminometer (Promega, Madison, WI, USA), and each value from the firefly luciferase construct was normalized by $\beta$-gal assay.

Western blot analysis. Total protein extracts were prepared in cell disruption buffer. The protein concentration was determined using the BCA standard curve method. Equal amounts of protein extract were separated by sodium dodecyl sulfate-polyacrylamide gel electrophoresis (SDS-PAGE) and transferred electrophoretically onto PVDF membranes. The membranes were blocked in 
A
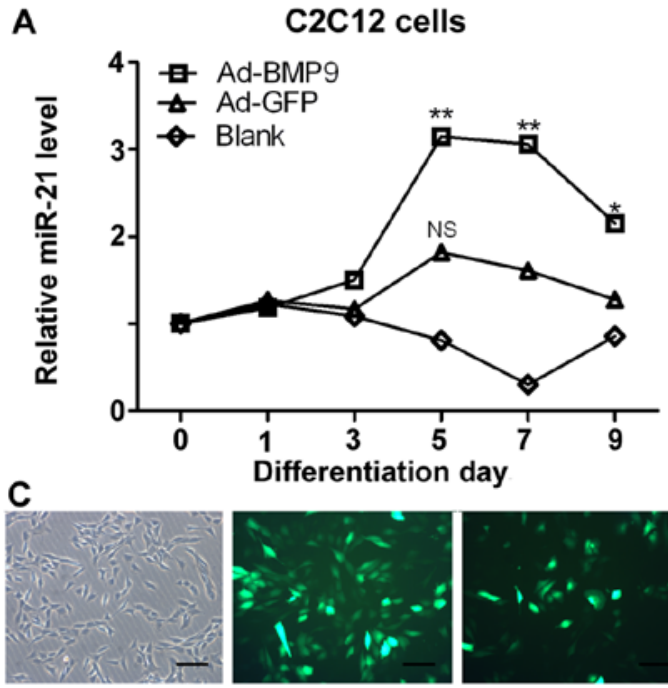

Blank

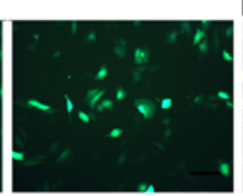

Ad-GFP

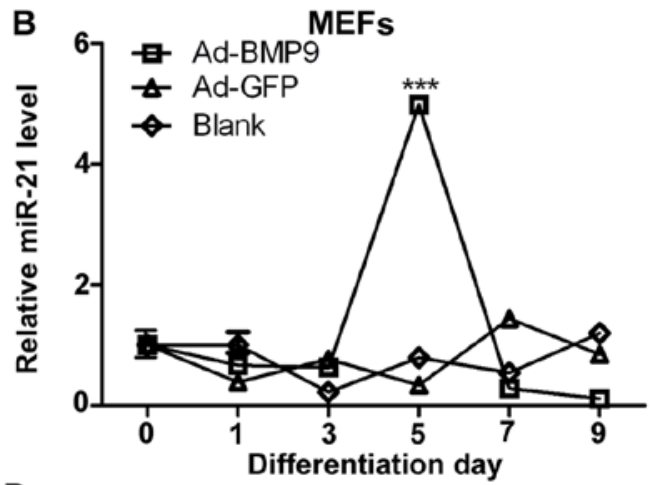

D
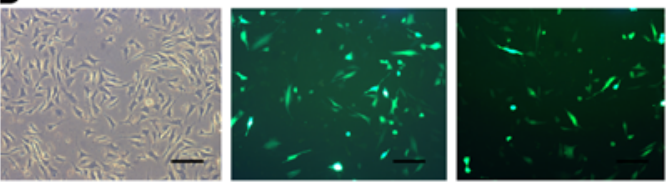

Blank

Ad-BMPg

Ad-GFP

$\mathbf{F}$

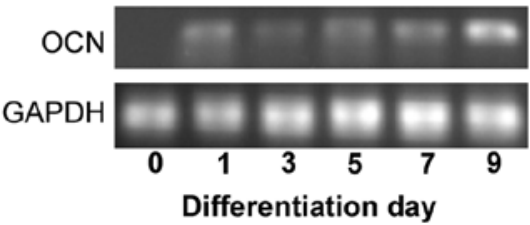

Figure 1. Upregulation of miR-21 is mediated by increased bone morphogenetic protein (BMP) 9 expression during the osteogenic differentiation of murine multilineage cells (MMCs). The expression levels of miR-21 were increased in the (A) C2C12 cells and (B) MEFs; this increase was induced by Ad-BMP9 or Ad-green fluorescent protein (GFP) [multiplicity of infection (MOI) $=5$, normalized to U6]. ${ }^{* *}$ p $<0.01$, ${ }^{* * *}$ p $<0.001$ vs. the value at day 0 . NS, no significance vs. the value at day 0 . Effectiveness of transfection of (C) C2C12 cells and (D) MEFs with Ad-BMP9 or Ad-GFP (MOI=5). Magnification, x100. White, white light used as the excitation light; green, blue light used as the excitation light. The transcription levels of osteocalcin (OCN) were analyzed by RT-qPCR in the (E) C2C12 cells and (F) MEFs during Ad-BMP9-induced osteogenic differentiation (normalized to GAPDH).

TBST containing $5 \%$ bovine serum albumin (BSA) at $37^{\circ} \mathrm{C}$ for $2 \mathrm{~h}$. The blocked membranes were probed with rabbit anti-Smad7 polyclonal antibody (1:1,000 dilution, Cat. no. ab124890; Abcam, Cambridge, MA, USA), rabbit anti-phosphorylated (p-)Smad1/5 monoclonalantibody (1:1,000 dilution Cat.no.9516; Cell Signaling Technology, Inc., Beverly, MA, USA), rabbit anti-Smad1/5/8 monoclonal antibody (1:1,000 dilution, Cat. no. sc-6031-R, Lot no. D1911; Santa Cruz Biotechnology, Inc., Santa Cruz, CA, USA), rabbit anti-runt-related transcription factor 2 (Runx2) monoclonal antibody (1:1,000 dilution, Cat. no. sc-10758, Lot no. A0810, Santa Cruz Biotechnology) or anti- $\beta$-actin mouse monoclonal antibody (1:1,000 dilution; Zhongshan Golden Bridge Biotechnology Co., Ltd., Beijing, China) overnight at $4^{\circ} \mathrm{C}$, and were subsequently washed with TBS containing $0.1 \%$ Tween-20. After being washed with TBST 3 times, with each wash lasting $15 \mathrm{~min}$, the membranes were incubated with goat anti-rabbit or goat anti-mouse horseradish peroxidaseconjugated secondary antibody (1:5,000 dilution; Zhongshan Golden Bridge Biotechnology Co., Ltd.), for $1 \mathrm{~h}$ at $37^{\circ} \mathrm{C}$. The secondary antibodies were detected with western chemiluminescence reagent (Millipore Corp., Billerica, MA, USA). The results were recorded using the Bio-Rad Electrophoresis Documentation system (Gel Doc 1000) and Quantity One software, version 4.5.0.

Statistical analysis. All quantitative experiments were performed in triplicate and/or repeated 3 times. Data are presented as the means + SD. Statistical significances between the control and treatment groups were determined by one-way analysis of variance and the Student's t-test. All statistical analyses were performed using GraphPad Prism 5 (GraphPad Software, Inc., La Jolla, CA, USA). P-values $<0.05,<0.01$ or $<0.001$ was considered to indicate statistically significant differences. NS represents no significant difference.

\section{Results}

Upregulation of $\mathrm{miR}-21$ is mediated by increased BMP9 expression during the osteogenic differentiation of MMCs. To identify the role which miR-21 plays during osteoblastic differentiation, we induced the osteogenic differentiation of $\mathrm{C} 2 \mathrm{C} 12$ cells (mouse precursor myoblasts) and MEFs by stimulating the cells with BMP9-conditioned medium. We measured the expression levels of miR-21 during the osteogenic differentiation of the $\mathrm{C} 2 \mathrm{C} 12$ cells and MEFs by RT-qPCR. We found that the expression of miR-21 increased on the 3rd day of differentiation, and peaked on the 5th day of differentiation (Ad-BMP9 group). The expression of miR-21 began to decrease from day 7 and on day 9, its expression was decreased (Fig. 1A). However, the expression of miR-21, in the control group (Ad-GFP; cells not infected with BMP9) was not significantly altered. Monitoring the expression of miR-21 in the MEFs confirmed the changes in miR-21 expression during osteogenic differentiation (Fig. 1B). Moreover, we showed that Ad-BMP9 and Ad-GFP, which both expressed the GFP marker, were effectively transfected into the $\mathrm{C} 2 \mathrm{C} 12$ cells and MEFs, as determined under a fluorescence microscope (Fig. 1C and D). 


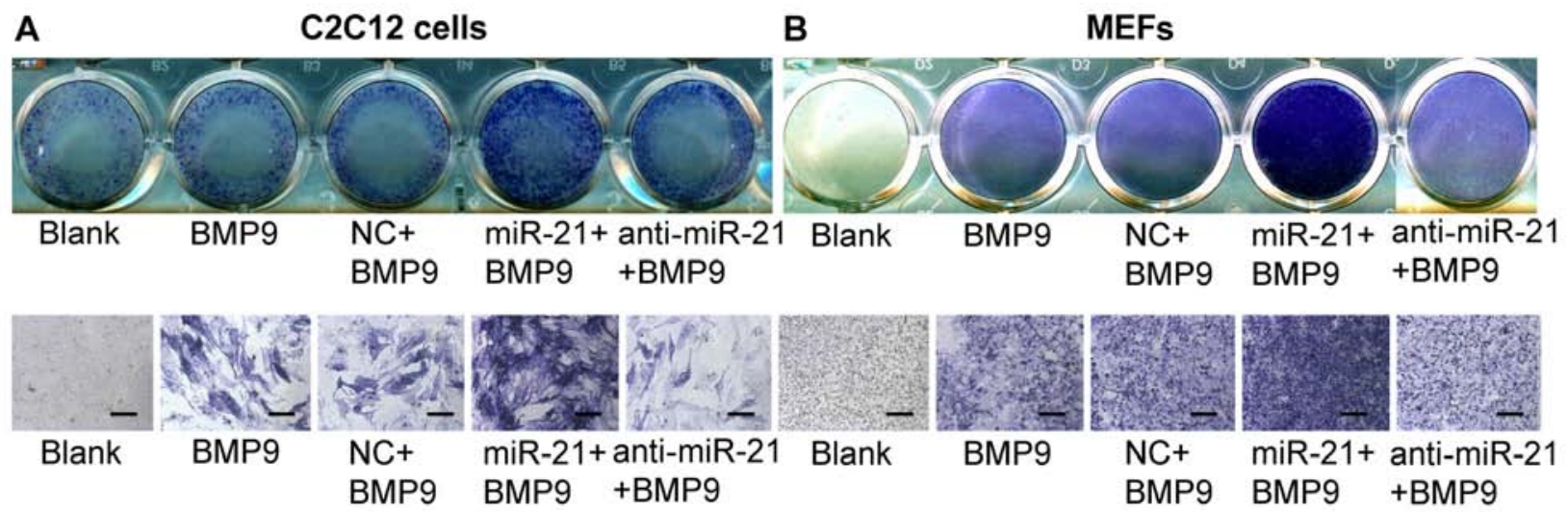

C

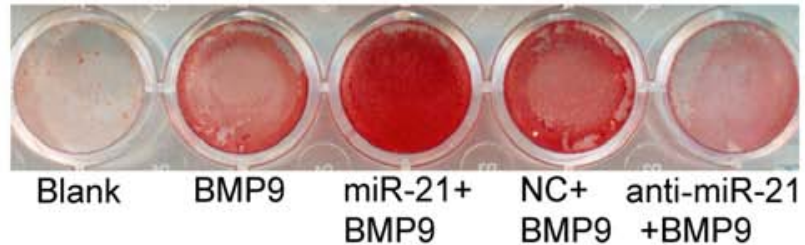

D

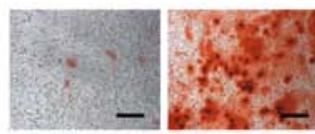

Blank BMP9

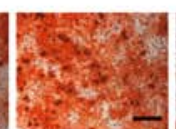

miR-21+ BMP9

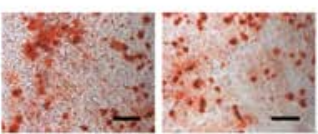

$\mathrm{NC}+$ anti-miR-21

BMP9 +BMP9
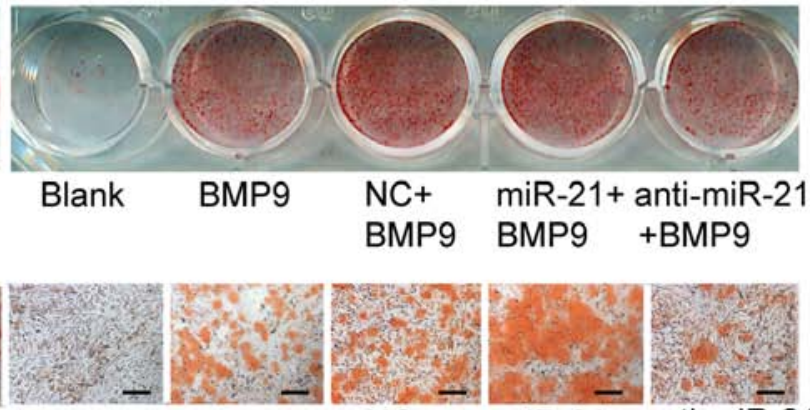

BMP9

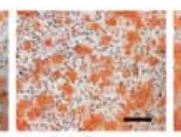

$\mathrm{NC}+$ BMP9

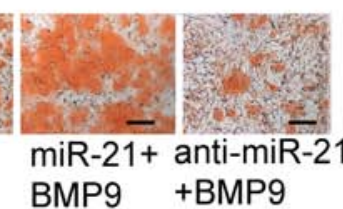

Figure 2. Overexpression of miR-21 enhances bone morphogenetic protein (BMP)9-induced alkaline phosphatase (ALP) activity and calcium deposition in murine multilineage cells (MMCs). miR-21 potentiates BMP9-induced ALP staining. (A) Subconfluent C2C12 cells and (B) MEFs were treated with green fluorescent protein (GFP)-conditioned medium (CM), BMP9-CM, miR.NC (100 nM) + BMP9-CM, miR-21 (100 nM) + BMP9-CM, or anti-miR-21 (100 nM) + BMP9-CM. The staining results were recorded at a lower (A and B, top panel) and higher magnification (A and B, bottom panel). ALP staining was conducted following 7 days of differentiation. miR-21 potentiates BMP9-induced matrix mineralization. (C) Subconfluent C2C12 cells and (D) MEFs were treated with GFP-CM, BMP9-CM, miR.NC (100 nM) + BMP9-CM, miR-21 (100 nM) + BMP9-CM, or anti-miR-21 (100 nM) + BMP9-CM. The Alizarin red S staining of matrix mineralization results were recorded at a lower $(\mathrm{C}$ and $\mathrm{D}$, top panel) and higher magnification ( $\mathrm{C}$ and $\mathrm{D}$, bottom panel). Alizarin red $\mathrm{S}$ staining was conducted following 14 days of differentiation. Each assay condition was done in triplicate. The results were repeated in at least 2 independent batches of experiments. NC, negative control. Lower magnification refers to cells in a 24-well plate being scanned under a scanner (ScanMaker 3600). Higher magnification refers to cells being scanned under a bright field microscope (x200).

Furthermore, we measured the osteocalcin (OCN; marker of osteogenic differentiation) expression levels by RT-qPCR, and the results revealed that the successful osteogenic differentiation of the $\mathrm{C} 2 \mathrm{C} 12$ cells and MEFs had occurred (Fig. 1E and F), as OCN expression increased with the induction of differentiation. We also compared the levels of endogenous miR-21 in the $\mathrm{C} 2 \mathrm{C} 12$ cells and MEFs by RT-qPCR. The miR-21 expression level was approximately 3 - and 5-fold higher compared to the control (Ad-GFP) on day 5 of differentiation in the $\mathrm{C} 2 \mathrm{C} 12$ cells and MEFs, respectively (Fig. 1A and B).

Overexpression of miR-21 increases BMP9-induced ALP and calcium deposition in MMCs. Subsequently, we examined the effect of exogenous miR-21 expression on BMP9-induced osteogenic differentiation. To achieve consistent and robust gene expression, miR-21 was overexpressed or inhibited using synthetic mmu-miR-21mimic (miR-21) or mmu-miR-21 inhibitor (anti-miR-21), respectively. The expression levels of miR-21 following transfection were verified by RT-qPCR (Fig. 3C and D). Whereas the exogenous expression of miR-21 alone did not induce any significant ALP activity (data not shown), the co-expression of BMP9 and miR-21 (miR-21 + BMP9 group) synergistically induced
ALP activity in the $\mathrm{C} 2 \mathrm{C} 12$ cells (Fig. 2A). Similar results were obtained with the MEFs (Fig. 2B). These results indicate that miR-21 potentiates the BMP9-induced osteoblast lineage commitment of MMCs.

We further analyzed the effect of miR-21 on late osteogenic differentiation by Alizarin red S staining of matrix mineralization. Whereas the exogenous expression of miR-21 alone did not induce any obvious calcium deposition (data not shown), the co-expression of BMP9 and miR-21 (miR-21 + BMP9 group) synergistically induced calcium deposition in the C2C12 cells (Fig. 2C). Similar results were obtained with the MEFs (Fig. 2D). These results indicate that miR-21 potentiates the BMP9-induced osteoblast lineage commitment of MMCs.

miR-21 increases BMP9/Smad signaling in MMCs. The BMP9/Smad signaling pathway plays a pivotal role in MSC differentiation (37). The positive correlation between BMP9-Smad signaling activity and miR-21 expression during the osteogenic differentiation of the $\mathrm{C} 2 \mathrm{C} 12$ cells and MEFs was revealed during our research. To determine whether the expression level of miR-21 affects Smad signaling activity during osteogenic differentiation, miR-21 was transfected into the $\mathrm{C} 2 \mathrm{C} 12$ cells and MEFs, and this was followed by 

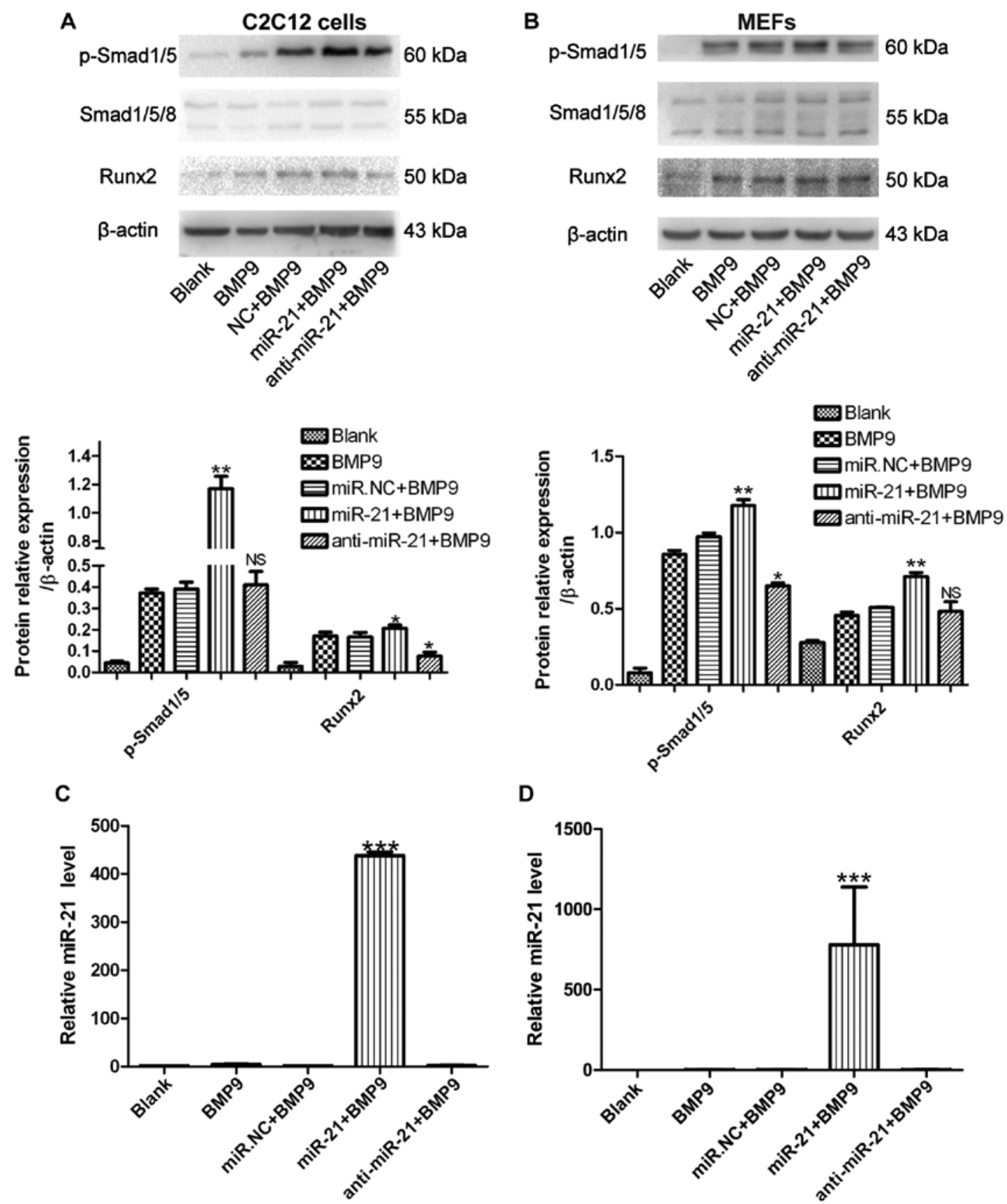

Figure 3. miR-21 increases bone morphogenetic protein (BMP)9-Smad signaling in murine multilineage cells (MMCs). (A) C2C12 cells and (B) MEFs were treated with green fluorescent protein (GFP)-conditioned medium (CM), BMP9-CM, miR.NC (500 nM) + BMP9-CM, miR-21 (500 nM) + BMP9-CM, or antimiR-21 (500 nM) + BMP9-CM, and phosphorylated (p-)Smad1/5, Smad1/5/8 and runt-related transcription factor 2 (Runx2) levels were measured by western blot analysis. $\beta$-actin was used as the internal control. ${ }^{* *} \mathrm{p}<0.01,{ }^{*} \mathrm{p}<0.5$ vs. miR.NC + BMP9. NS, not significant vs. miR.NC + BMP9. (C) C2C12 cells and (D) MEFs were treated with GFP-CM, BMP9-CM, miR.NC (500 nM) + BMP9-CM, miR-21 (500 nM) + BMP9-CM, or anti-miR-21 (500 nM) + BMP9-CM, and RT-qPCR analysis of miR-21 expression in the $\mathrm{C} 2 \mathrm{C} 12$ cells and MEFs was conducted. ${ }^{* * *}$ p $<0.001$ vs. BMP9. NC, negative control.

RT-qPCR analysis. Increased levels of miR-21 [approximately 700- and 400-fold greater compared to the endogenous miR-21 levels (BMP9 group)] were expressed in the $\mathrm{C} 2 \mathrm{C} 12$ cells and MEFs, respectively (Fig. 3C and D). We then measured the expression levels of $\mathrm{p}-\mathrm{Smad} 1 / 5$ and Runx 2 by western blot analysis. Compared with the negative control (NC), during osteogenesis induced by BMP9, the levels of p-Smad1/5 and Runx 2 were upregulated. However, in the C2C12 cells transfected with anti-miR-21 (anti-miR-21 + BMP9), p-Smad1/5 expression did not differ significantly compared to the $\mathrm{NC}$ and
Runx2 expression was decreased compared to the $\mathrm{NC}$; in the MEFs transfected with anti-miR-21 (anti-miR-21 + BMP9), $\mathrm{p}-\mathrm{Smad} 1 / 5$ expression was decreased compared to the NC and Runx 2 expression did not differ significantly compared to the NC (Fig. 3A and B). These data indicated that the ectopic expression of miR-21 promoted $\mathrm{p}-\mathrm{Smad} 1 / 5$ expression during the osteogenic differentiation of MMCs. We also observed that Runx 2 expression tended to be elevated when the cells were transfected with miR-21 during osteogenesis induced by BMP9. However, the inhibition of miR-21 (with anti-miR-21) 

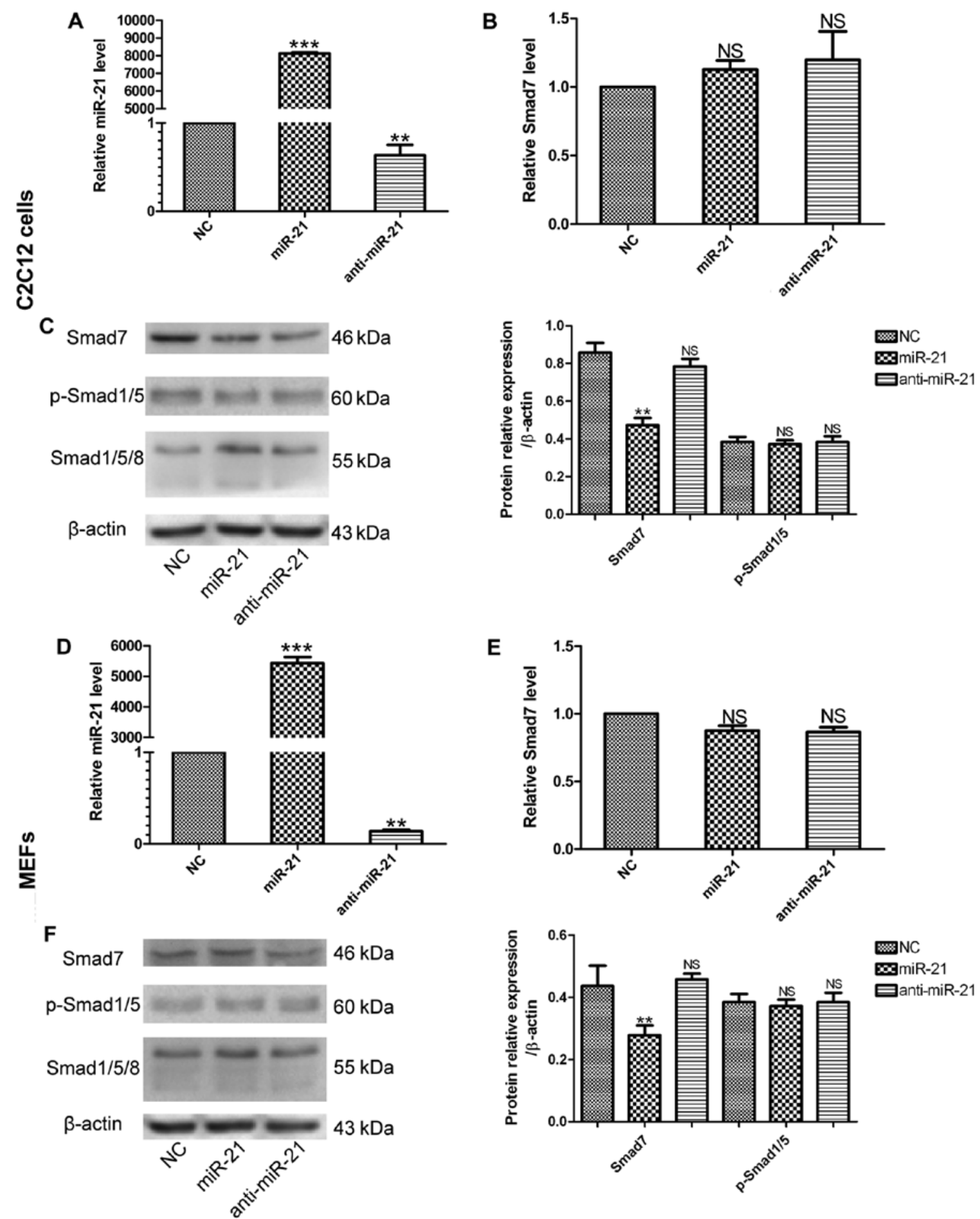

Figure 4. miR-21 regulates expression of Smad7. (A) C2C12 cells and (D) MEFs were treated with miR.NC (500 nM), miR-21 (500 nM), anti-miR-21 (500 nM), Analysis of miR-21 expression in each group by RT-qPCR. ${ }^{* * *} \mathrm{p}<0.01$ and ${ }^{* * *} \mathrm{p}<0.001$ vs. negative control (NC). (B) C2C12 cells and (E) MEFs: analysis of Smad7 epxression by RT-qPCR. NS, not significant. (C) C2C12 cells and (F) MEFs: the levels of Smad7, phosphorylated (p-)Smad1/5 and Smad1/5/8 were measured by western blot analysis. $\beta$-actin was used as the internal controls. ${ }^{* *} \mathrm{p}<0.01$ vs. negative control (NC). NS, not significant vs. NC.

had an almost reverse effect during osteogenic differentiation. We found that the inhibition of miR-21 by transfection with anti-miR-21 only slightly affected BMP9-Smad signaling, whereas the enhancement of miR-21 had a marked effect on Smad signaling. These data indicate that a high expression level of miR-21 is significant for sustaining BMP9-Smad signaling activity during osteogenesis.
miR-21 regulates the expression of Smad7. To investigate the downstream targets underlying the regulation of MSC differentiation by miR-21, we used a bioinformatics approach and summarized the context of three widely used miRNA databases, TargetScan, PicTar and miRanda, to identify the target genes. Bioinformatics prediction targets are usually categorized by specified cellular function. The majority of the 

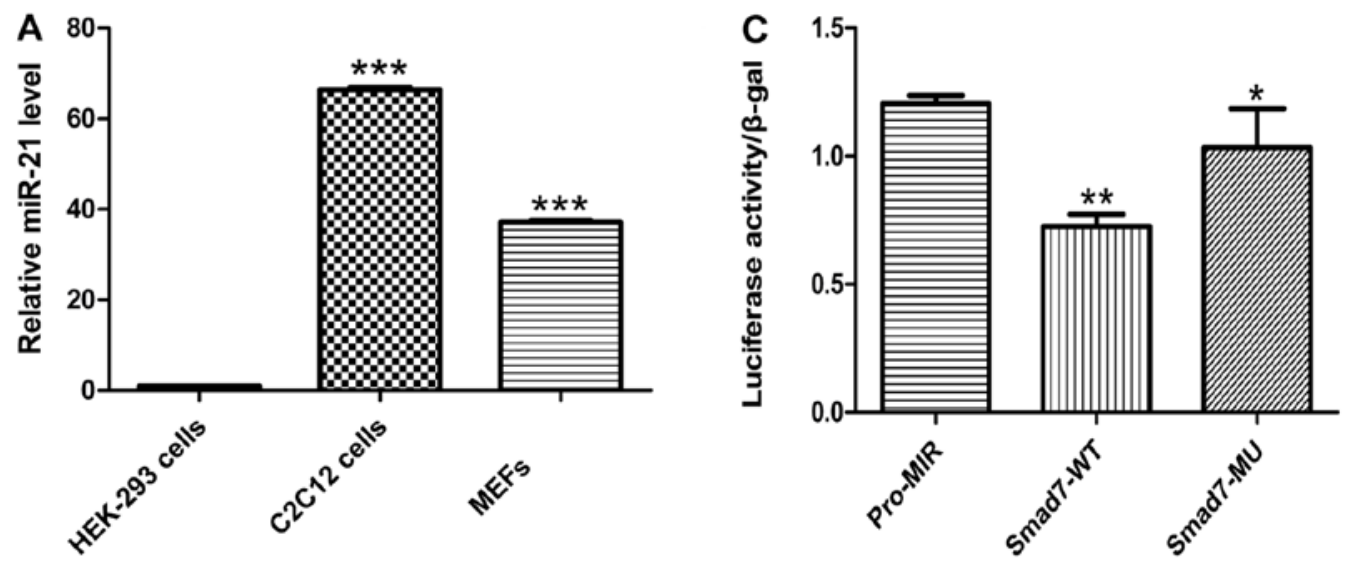

B Smad7 3'UTR wild-type

MiR-21

5'... UGUUUAGAAUUUAACAUAAGCUA....'

3' AGUUGUAGUCAGACUAUUUCGAU 5'

| I|

Smad7 3'UTR mutant-type 5'...UGUUUAGAAUUUAACATATCGAA... 3'

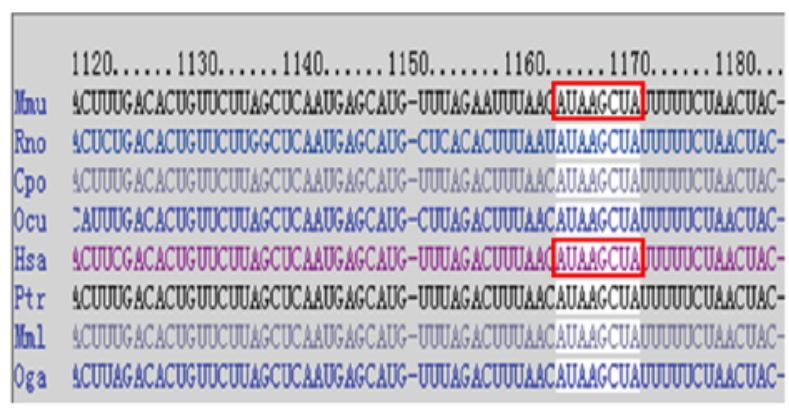

Figure 5. miR-21 targets the 3'-UTR of Smad7 mRNA. (A) RT-qPCR of miR-21 expression in HEK-293, C2C12 cells and MEFs. (B) The sequence of miR-21 complementation with Smad7 3'-UTR wild-type and mutant-type reporter. In mice and humans, miR-21 complementation with Smad7 3'-UTR wild-type is shown. (C) The Smad7 3'-UTR wild-type (200 ng) and mutant-type (200 ng) reporter and pMIR-Cont (200 ng) were co-transfected with miR-21 (100 nM) into HEK-293 cells. After $48 \mathrm{~h}$, luciferase activities were measured. The data shown represent the means + SD; ${ }^{*} \mathrm{p}<0.5,{ }^{* *} \mathrm{p}<0.01$ and ${ }^{* * *} \mathrm{p}<0.001$ vs. Pro-MIR.

miR-21 targets predicted by these databases are involved in the BMP9-Smad signaling pathway, and our results demonstrated that miR-21 affected BMP9-Smad signaling activity during the osteogenic differentiation of MMCs. We hypothesized that the desired target of miR-21 would be more robustly expressed in a less-differentiated cell population. The target gene is likely to interfere with the events that are essential to cell fate or the initiation of differentiation. Based on these considerations, we selected Smad7 as the miR-21 target candidate. Smad7 is a negative regulator of the BMP9/Smad signaling pathway (29). To determine whether Smad7 is a direct target of miR-21 during the osteogenic differentiation of MMCs, we measured the mRNA and protein expression levels of Smad7 following the regulation of miR-21 expression by transfecting the MMCs with miR-21, NC or anti-miR-21. After confirming the transfection efficiency (Fig. 4A and D), we found that in the miR-21transfected cells, the protein levels of Smad7 were decreased compared with the negative control (NC) during osteogenesis induced by BMP9, whereas the transfection of the MMCs with anti-miR-21 did not markedly increase the protein level of Smad7 compared with the NC (Fig. 4C and F). As regards the mRNA expression of Smad7, transfection with miR-21 did not induce any significant change, and moreover, transfection with anti-miR-21 also had no significant effect on Smad7 mRNA expressoin (Fig. 4B and E). We hypothesized that this may be attributed to the fairly high concentration of endogenous miR-21, which was too high to be offset by anti-miR-21. We also measured the protein levels of $\mathrm{p}$-Smad1/5 and Smad1/5/8, and we found that the levels in the 3 groups did not differ significantly (Fig. 4C and F). These results demonstrated that miR-21 significantly decreased the protein expression level of Smad7, but did not markedly alter its mRNA expression level, as compared with the control (NC) group. Moreover, the up- or downregulation of miR-21 alone did not have a significant effect on the p-Smad1/5 levels in either of the two cell lines.

miR-21 targets the 3'-UTR of Smad7 mRNA. Since the site at which miR-21 binds to the 3'-UTR of Smad7 was already verified using target prediction tools (TargetScan, PicTar and miRanda), we sought to determine whether Smad7 is a target of miR-21 using luciferase reporter assay. The Smad7 gene contains the same sequence at the 3'-UTR, which is complementary to the miR-21 seed sequence (MRE). We directly generated matching miR-21 target sites and cloned these wild-type and mutant sites into the multiple cloning sites in the pMIR-REPORT luciferase, named Smad7-WT and Smad7-MU (Fig. 5B). We found that the ectopic expression of miR-21 by co-transfection of miR-21 and the pMIR-REPORTSmad7 vector into HEK-293 cells suppressed the activity of Renilla luciferase. Notably, the HEK-293 cells did not exhibit 
endogenous miR-21 expression (Fig. 5A). miR-21 decreased the luciferase activity of Smad7-WT, but not that of Smad7-MU, and thus these results confirmed the direct interaction of miR-21 with the Smad7 3'-UTR (Fig. 5C).

\section{Discussion}

Bone diseases, which are characterized by decreased bone mass and the microarchitectural deterioration of bone tissue, represent an increasing medical and socioeconomic burden. We have identified BMP9 has previously been identified as one of the most robust osteogenic BMPs, both in vitro and in vivo $(25,27,30-37)$. As one of the most extensively studied BMPs, BMP9 may exert its signaling activity by regulating a distinct set of downstream mediators, including miRNAs, in MMCs. Although BMP9 has been demonstrated to be one of the most potent osteogenic BMPs, relatively little is known about the specific mechanisms responsible for its potency. Therefore, the exact roles which miRNAs play in BMP9-induced osteogenic signaling remain to be elucidated.

The BMP9/Smad signaling pathway plays an important role in skeletal development, bone formation and stem cell differentiation. Upon binding specific cell-surface receptor kinases, BMP-mediated signal transduction begins with the phosphorylation of Smads and subsequent heterodimer formation. Lamplot et al demonstrated that, similar to other osteogenic BMPs, BMP9 promotes the activation of Smad1/5/8 (38). miRNAs are endogenous modulation factors which can precisely regulate signal transduction in a time- and dosage-dependent manner. miR-21 synergizes with BMP9 and influences this process by modulating the interaction of Smad7 and BMP9/Smad signaling to control the duration and magnitude of the $\mathrm{p}-\mathrm{Smad} 1 / 5 / 8$ cascade. However, in our study, the exogenous expression of miR-21 alone did not change the expression of p-Smad1/5/8 in MMCs (Fig. 4C and F).

Smad7 belongs to the group of antagonistic/inhibitory Smads (I-Smad), and Smad7 or dorsomorphin has been suggested to prevent BMP signaling in a study using mutant activin receptor-like 2 (ALK2) in fibrodysplasia ossificans progressiva (FOP) (39). Of note, Smad7 contains the miR-21 binding site, which is complementary to the miR-21 seed sequence in the 3'-UTR. We speculated in the present study that miR-21 and Smad7 interaction may fine-tune BMP9/Smad signaling activity and gene-regulation networks during MMCs ostegenic differentiation. miR-21 can decrease Smad7 thus affecting p-Smad1/5, and fine-tuning BMP9/Smad signaling activity.

Our results demonstrated that miR-21 expression was upregulated during the osteogenic differentiation of MMCs (Fig. 1A and B). Previous research has demonstrated that the BMP9/Smad signaling pathway plays a critical role in MSC differentiation, and its activation is sustained during this process (40). We suggest that miR-21 reduced Smad7 levels to maintain BMP9/Smad signaling activation during the osteogenic differentiation process. The balance of miR-21 and Smad7 expression could fine-tune the duration and magnitude of BMP9/Smad signaling activity to determine cell fate (41). It has been determined that BMP9-induced miR-21 upregulation was one of the mechanisms through which BMP9 contributes to bone formation (42). Further studies are, however, required to validate other predicted targets involved in bone development. Nonetheless, our findings indicate a novel mechanism through which enhanced BMP9-induced osteoblastic bone formation occurs via the upregulation of miR-21 expression in MMCs.

\section{Acknowledgements}

The authors would like to thank T.C. He (Medical Center, The University of Chicago) for his kind provision of the recombinant adenovirus (Ad-BMP9 and AdGFP).

\section{References}

1. Wang X, Guo B, Li Q, Peng J, Yang Z, Wang A, Li D, Hou Z, Lv K, Kan G, et al: miR-214 targets ATF4 to inhibit bone formation. Nat Med 19: 93-100, 2013.

2. Lewis BP, Burge CB, and Bartel DP: Conserved seed pairing, often flanked by adenosines, indicates that thousands of human genes are microRNA targets. Cell 120: 15-20, 2005.

3. Doench JG and Sharp PA: Specificity of microRNA target selection in translational repression. Genes Dev 18: 504-511, 2004.

4. Eskildsen T, Taipaleenmäki H, Stenvang J, Abdallah BM, Ditzel N, Nossent AY, Bak M, Kauppinen S and Kassem M: MicroRNA-138 regulates osteogenic differentiation of human stromal (mesenchymal) stem cells in vivo. Proc Natl Acad Sci USA 108: 6139-6144, 2011.

5. Dong J, Cui X, Jiang Z and Sun J: MicroRNA-23a modulates tumor necrosis factor-alpha-induced osteoblasts apoptosis by directly targeting Fas. J Cell Biochem 114: 2738-2745, 2013.

6. Hassan MQ, Maeda Y, Taipaleenmaki H, Zhang W, Jafferji M, Gordon JA, Li Z, Croce CM, van Wijnen AJ, Stein JL, et al: miR-218 directs a Wnt signaling circuit to promote differentiation of osteoblasts and osteomimicry of metastatic cancer cells. J Biol Chem 287: 42084-42092, 2012.

7. Hu R, Li H, Liu W, Yang L, Tan YF and Luo XH: Targeting miRNAs in osteoblast differentiation and bone formation. Expert Opin Ther Targets 14: 1109-1120, 2010.

8. Inose H, Ochi H, Kimura A, Fujita K, Xu R, Sato S, Iwasaki M, Sunamura S, Takeuchi Y, Fukumoto S, et al: A microRNA regulatory mechanism of osteoblast differentiation. Proc Natl Acad Sci USA 106: 20794-20799, 2009.

9. Li H, Xie H, Liu W, Hu R, Huang B, Tan YF, Xu K, Sheng ZF, Zhou HD, Wu XP and Luo XH: A novel microRNA targeting HDAC5 regulates osteoblast differentiation in mice and contributes to primary osteoporosis in humans. J Clin Invest 119: 3666-3677, 2009.

10. Busacca S, Germano S, De Cecco L, Rinaldi M, Comoglio F, Favero F, Murer B, Mutti L, Pierotti M and Gaudino G: MicroRNA signature of malignant mesothelioma with potential diagnostic and prognostic implications. Am J Respir Cell Mol Biol 42: 312-319, 2010.

11. Chan JA, Krichevsky AM and Kosik KS: MicroRNA-21 is an antiapoptotic factor in human glioblastoma cells. Cancer Res 65 : 6029-6033, 2005.

12. Chang SS, Jiang WW, Smith I, Poeta LM, Begum S, Glazer C, Shan S, Westra W, Sidransky D and Califano JA: MicroRNA alterations in head and neck squamous cell carcinoma. Int $\mathrm{J}$ Cancer 123: 2791-2797, 2008.

13. Hatley ME, Patrick DM, Garcia MR, Richardson JA, Bassel-Duby R, van Rooij E and Olson EN: Modulation of K-Ras-dependent lung tumorigenesis by MicroRNA-21. Cancer Cell 18: 282-293, 2010.

14. Krichevsky AM and Gabriely G: miR-21: a small multi-faceted RNA. J Cell Mol Med 13: 39-53, 2009.

15. Ladeiro Y, Couchy G, Balabaud C, Bioulac-Sage P, Pelletier L, Rebouissou S and Zucman-Rossi J: MicroRNA profiling in hepatocellular tumors is associated with clinical features and oncogene/tumor suppressor gene mutations. Hepatology 47: 1955-1963, 2008.

16. Moriyama T, Ohuchida K, Mizumoto K, Yu J, Sato N, Nabae T, Takahata S, Toma H, Nagai E and Tanaka M: MicroRNA-21 modulates biological functions of pancreatic cancer cells including their proliferation, invasion, and chemoresistance. Mol Cancer Ther 8: 1067-1074, 2009.

17. Si ML, Zhu S, Wu H, Lu Z, Wu F and Mo YY: miR-21-mediated tumor growth. Oncogene 26: 2799-2803, 2007. 
18. Zhu S, Si ML, Wu H and Mo YY: MicroRNA-21 targets the tumor suppressor gene tropomyosin 1 (TPM1). J Biol Chem 282: 14328-14336, 2007.

19. Zhu S, Wu H, Wu F, Nie D, Sheng S and Mo YY: MicroRNA-21 targets tumor suppressor genes in invasion and metastasis. Cell Res 18: 350-359, 2008.

20. Li Z, Hassan MQ, Volinia S, van Wijnen AJ, Stein JL, Croce CM, Lian JB and Stein GS: A microRNA signature for a BMP2-induced osteoblast lineage commitment program. Proc Natl Acad Sci USA 105: 13906-13911, 2008.

21. Itoh T, Takeda S and Akao Y: MicroRNA-208 modulates BMP-2-stimulated mouse preosteoblast differentiation by directly targeting V-ets erythroblastosis virus E26 oncogene homolog 1. J Biol Chem 285: 27745-27752, 2010.

22. Kuree J, Dixit M, Tyagi AM, Mansoori MN, Srivastava K, Raghuvanshi A, Maurya R, Trivedi R, Goel A and Singh D: miR-542-3p suppresses osteoblast cell proliferation and differentiation, targets BMP-7 signaling and inhibits bone formation. Cell Death Dis: February 6, 2014 (Epub ahead of print) doi: 10.1038 /cddis.2014.4.

23. Mei Y, Bian C, Li J, Du Z, Zhou H, Yang Z and Zhao RC: miR-21 modulates the ERK-MAPK signaling pathway by regulating SPRY2 expression during human mesenchymal stem cell differentiation. J Cell Biochem 114: 1374-1384, 2012.

24. Yang N, Wang G, Hu C, Shi Y, Liao L, Shi S, Cai Y, Cheng S, Wang X, Liu Y, et al: Tumor necrosis factor $\alpha$ suppresses the mesenchymal stem cell osteogenesis promoter miR-21 in estrogen deficiency-induced osteoporosis. J Bone Miner Res 28: 559-574, 2013.

25. Wang RN, Green J, Wang Z, Deng Y, Qiao M, Peabody M, Zhang Q, Ye J, Yan Z, Denduluri S, et al: Bone Morphogenetic Protein (BMP) signaling in development and human diseases. Genes Dis 1: 87-105, 2014.

26. Min S, Liang X, Zhang M, Zhang Y, Mei S, Liu J, Liu J, Su X, Cao S, Zhong X, et al: Multiple tumor-associated microRNAs modulate the survival and longevity of dendritic cells by targeting YWHAZ and Bcl2 signaling pathways. J Immunol 190: 2437-2446, 2013.

27. Tang N, Song W-X, Luo J, Luo X, Chen J, Sharff KA, Bi Y, He BC, Huang JY, Zhu GH, et al: BMP-9-induced osteogenic differentiation of mesenchymal progenitors requires functional canonical Wnt/ $\beta$-catenin signalling. J Cell Mol Med 13 (8B) 2448-2464, 2009.

28. Amin S, Riggs BL, Melton LJ III, Achenbach SJ, Atkinson EJ and Khosla S: High serum IGFBP-2 is predictive of increased bone turnover in aging men and women. J Bone Miner Res 22. 799-807, 2007

29. Zhou H,Zou S, Lan Y, Fei W, Jiang R and Hu J: Smad7 modulates TGF $\beta$ signaling during cranial suture development to maintain suture patency. J Bone Miner Res 29: 716-724, 2014

30. Luther G, Wagner ER, Zhu G, Kang Q, Luo Q, Lamplot J, Bi Y, Luo X, Luo J, Teven C, et al: BMP-9 induced osteogenic differentiation of mesenchymal stem cells: molecular mechanism and therapeutic potential. Curr Gene Ther 11: 229-240, 2011.
31. Cheng H, Jiang W, Phillips FM, Haydon RC, Peng Y, Zhou L, Luu HH, An N, Breyer B, Vanichakarn P, et al: Osteogenic activity of the fourteen types of human bone morphogenetic proteins (BMPs). J Bone Joint Surg Am 85-A: 1544-1552, 2003.

32. Kang Q, Sun MH, Cheng H, Peng Y, Montag AG, Deyrup AT, Jiang W, Luu HH, Luo J, Szatkowski JP, et al: Characterization of the distinct orthotopic bone-forming activity of 14 BMPs using recombinant adenovirus-mediated gene delivery. Gene Ther 11: 1312-1320, 2004

33. Peng Y, Kang Q, Cheng H, Li X, Sun MH, Jiang W, Luu HH, Park JY, Haydon RC and He TC: Transcriptional characterization of bone morphogenetic proteins (BMPs)-mediated osteogenic signaling. J Cell Biochem 90: 1149-1165, 2003.

34. Peng Y, Kang Q, Luo Q, Jiang W, Si W, Liu BA, Luu HH, Park JK, Li X, Luo J, et al: Inhibitor of DNA binding/differentiation helix-loop-helix proteins mediate bone morphogenetic protein-induced osteoblast differentiation of mesenchymal stem cells. J Biol Chem 279: 32941-32949, 2004

35. Luo Q, Kang Q, Si W, Jiang W, Park JK, Peng Y, Li X, Luu HH, Luo J, Montag AG, et al: Connective tissue growth factor (CTGF) is regulated by Wnt and bone morphogenetic proteins signaling in osteoblast differentiation of mesenchymal stem cells. J Biol Chem 279: 55958-55968, 2004.

36. Sharff KA, Song WX, Luo X, Tang N, Luo J, Chen J, Bi Y, He BC, Huang J, Li X, et al: Heyl basic helix-loop-helix protein plays an important role in mediating BMP9-induced osteogenic differentiation of mesenchymal progenitor cells. J Biol Chem 284: 649-659, 2009.

37. Luu HH, Song WX, Luo X, Manning D, Luo J, Deng ZL, Sharff KA, Montag AG, Haydon RC and He TC: Distinct roles of bone morphogenetic proteins in osteogenic differentiation of mesenchymal stem cells. J Orthop Res 25: 665-677, 2007.

38. Lamplot JD, Qin J, Nan G, Wang J, Liu X, Yin L, Tomal J, Li R, Shui $\mathrm{W}$, Zhang $\mathrm{H}$, et al: BMP9 signaling in stem cell differentiation and osteogenesis. Am J Stem Cells 2: 1-21, 2013.

39. Yu PB, Deng DY, Lai CS, Hong CC, Cuny GD, Bouxsein ML, Hong DW, McManus PM, Katagiri T, Sachidanandan C, et al: BMP type I receptor inhibition reduces heterotopic ossification. Nat Med 14: 1363-1369, 2008.

40. Augello A and De Bari C: The regulation of differentiation in mesenchymal stem cells. Hum Gene Ther 21: 1226-1238, 2010.

41. Li H, Yang F, Wang Z, Fu Q and Liang A: MicroRNA-21 promotes osteogenic differentiation by targeting small mothers against decapentaplegic 7. Mol Med Rep 12: 1561-1567, 2015.

42. Yang N, Wang G, Hu C, Shi Y, Liao L, Shi S, Cai Y, Cheng S, Wang X, Liu Y, et al: Tumor necrosis factor $\alpha$ suppresses the mesenchymal stem cell osteogenesis promoter miR-21 in estrogen deficiency-induced osteoporosis. J Bone Miner Res 28 : $559-573,2013$ 